\title{
Un raro caso di Herpes Zoster complicato da paralisi asintomatica del nervo frenico in un paziente in emodialisi
}

\author{
Marco Gallo, Sandro Bandini, Giorgio Monzani, Daniela Sacchi \\ Centro Dialisi Ulivella, Istituto Fiorentino di Cura e Assistenza, Firenze
}

\begin{abstract}
An unusual case of Herpes Zoster complicated by asymptomatic phrenic nerve paralysis in a hemodialysis patient Herpes Zoster ( $\mathrm{HZ}$ ) is the clinical manifestation of latent Varicella Zoster Virus (VZV) reactivation which can occur several decades after the early infection by Varicella Virus. Herpes Zoster usually affects the sensory neurons but may occasionally involve motor neurons corresponding to the dermatomes affected by skin lesions, resulting in flaccid paralysis of their muscles. Phrenic nerve paralysis is commonly caused by surgical or traumatic injuries, malignant tumors, neurodegenerative disorders or idiopathic diseases. However, in rare cases, it has been reported a diaphragmatic paralysis resulting from infection by Herpes Varicella Zoster Virus (VZV). We report a case of a 58 years old man, undergoing hemodialysis, who developed a left diaphragmatic paralysis after a typical Herpes Zoster rash involving the dermatome C3-C4 on the left side. The clinical and radiological results revealed no local causes of injury to the phrenic nerve. Hemidiaphragmatic paralysis was probably related to the infection by VZV. We asked ourselves if underdosing of the antiviral therapy (in the uremic patient) or the shortness of the treatment may have contributed to the development of this complication. However we believe that the risks resulting from overdosing outweigh the benefits. Therefore further studies, especially in ESRD, would be needed in order to evaluate if the dose adjustment and/or a prolongation of the antiviral therapy may have a protective effect on the onset of diaphragmatic paralysis.
\end{abstract}

Keywords: Diaphragmatic paralysis, Phrenic nerve paralysis, Hemodialysis, Herpes Zoster, Shingles

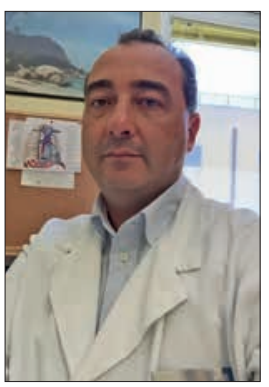

Marco Gallo

\section{Introduzione}

L'Herpes Zoster è la manifestazione clinica della riattivazione del Virus Varicella Zoster (VZV) latente, che può verificarsi anche diversi decenni dopo aver contratto la Varicella. L'Herpes Zoster solitamente colpisce i neuroni sensitivi ma, occasionalmente, può coinvolgere i motoneuroni corrispondenti ai dermatomeri interessati dalla lesione cutanea,

Accepted: August 27, 2015

Published online: October 12, 2015

Indirizzo per la corrispondenza:

Dr. Marco Gallo

Centro Dialisi Ulivella

Istituto Fiorentino di Cura e Assistenza

Via del Pergolino 4/6

50139 Firenze

marco.gallo@tin.it

con conseguente paralisi flaccida dei relativi muscoli. Dai neuroni sensitivi primitivi gangliari (dove rimane latente fino alla sua riattivazione) il Virus Varicella Zoster può diffondersi lungo l'assone, oltre che in direzione centrifuga, verso la cute del dermatomero, anche in direzione centripeta, fino a poter invadere il midollo spinale e a poter produrre, a seconda delle strutture interessate, manifestazioni cliniche che possono variare da paresi segmentali (per diffusione virale alle sole corrispondenti corna anteriori) fino a quadri più o meno estesi di mielite trasversa. Tale meccanismo spiegherebbe perché, in casi di Herpes Zoster con coinvolgimento motorio, alcuni Autori abbiano rilevato alterazioni elettromiografiche frequentemente non confinate al segmento invaso dal rash cutaneo ma più estese, sia in senso cranio-caudale che a livello controlaterale (1).

Il coinvolgimento del nervo frenico con successiva paralisi diaframmatica può portare a disfunzioni respiratorie ed è comunemente causato da lesioni chirurgiche e traumatiche, da neoplasie maligne e da malattie neurodegenerative (2). Esistono anche forme di paralisi idiopatica del diaframma che alcuni Autori sospettano essere correlate a infezioni virali (3). L'associazione della paralisi causata dal coinvolgimento del nervo frenico nel corso di svariate infezioni virali è una condi- 


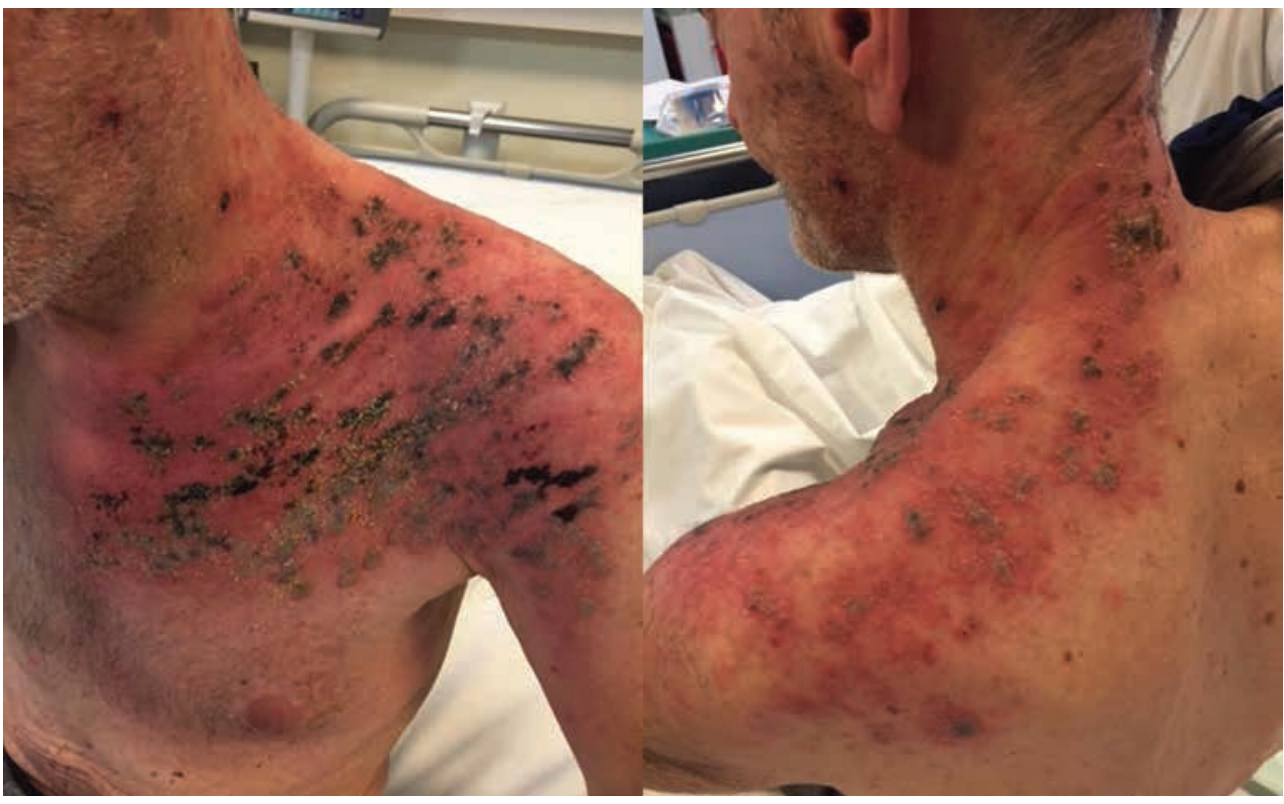

Fig. 1 - Eritema vescicoloso da Herpes Zoster riguardante i dermatomeri C3C4 di sinistra.

zione rara ma descritta (4). D'altra parte, l'infezione da Virus Herpes Varicella Zoster (VZV) è una malattia comune, ed è ben noto che si manifesta con un eritema vescicoloso a distribuzione dermatomerica unilaterale, solitamente associato a nevralgie neurosensitive. I due emidiaframmi, di destra e di sinistra, sono innervati separatamente, con innervazione motoria data esclusivamente dal nervo frenico (ramo del plesso cervicale che ha origine dalle radici spinali di C3-C4-C5) e innervazione sensitiva data congiuntamente dal nervo frenico e dai nervi intercostali inferiori. Dopo la sua origine, il nervo frenico discende nel collo e, passando fra l'arteria e la vena succlavia, penetra nel torace lateralmente al nervo vago. II nervo di destra passa lateralmente alla vena brachio-cefalica e alla vena cava superiore, mentre quello di sinistra incrocia l'arco dell'aorta; entrambi passano davanti all'ilo polmonare e, giunti sul diaframma, si distribuiscono al muscolo e alla pleura diaframmatica.

L'interessamento del plesso cervicale, in corso di infezione da VZV, può causare disfunzione del nervo frenico o paralisi diaframmatica (5). Sono stati pubblicati pochi casi, nella popolazione generale, di paralisi emidiaframmatica associata a infezione da Herpes Zoster (6-16). Da una revisione effettuata sul motore di ricerca Pubmed (http://www. ncbi.nlm.nih.gov/pubmed), non risulta alcun caso segnalato nella popolazione uremica. Dal momento che la paralisi diaframmatica unilaterale è spesso asintomatica e misconosciuta, è probabile che questa sia più comune di quanto riportato in letteratura.

Riportiamo il caso di un uomo con paralisi emidiaframmatica sinistra successiva alla comparsa di una tipica eruzione cutanea di tipo vescicoloso da Herpes Zoster a carico del dermatomero C3-C4 omolaterale.

\section{Caso clinico}

Uomo di 58 anni, affetto da artrite reumatoide, mieloma multiplo iposecernente (IgG k), diabete mellito insulino-dipendente e malattia renale cronica da nefropatia vascolare in trattamento emodialitico trisettimanale da circa 8 mesi. Il paziente era sottoposto a somministrazione di farmaci abitualmente utilizzati a fine dialisi (Epoetina beta $6000 \mathrm{UI} / \mathrm{sett}$, Ferro trivalente $62.5 \mathrm{mg} / \mathrm{sett}$, Calcio levofolinato $25 \mathrm{mg} / \mathrm{sett}$, Cianocobalamina 1000 mcg/sett e Carnitina $3 \mathrm{~g} / \mathrm{sett}$ ) oltre alla terapia domiciliare, che comprendeva Insulina lispro $4 \mathrm{UI} \times 3$, Bisoprololo $2.5 \mathrm{mg}$, Gabapentin $100 \mathrm{mg}$, Sertralina $50 \mathrm{mg}$, Pantoprazolo $20 \mathrm{mg}$, Metilprednisolone $8 \mathrm{mg}$ e Lorazepam $1 \mathrm{mg}$. Prima del trasferimento presso il nostro centro dialisi, il paziente è stato ricoverato per scompenso cardiaco da grave disfunzione sistolica e diastolica di tipo restrittivo con severa depressione della funzione sistolica (FE 32\%). Nel sospetto di amiloidosi, è stato sottoposto a esami mirati (biopsia renale e del grasso periombelicale) che non hanno evidenziato alcuna positività. Sempre nel corso del medesimo ricovero, è stato riscontrato un massivo versamento pleurico bilaterale che ha richiesto, dopo il posizionamento di un pig-tail toracico nel cavo pleurico destro, ripetuti drenaggi di liquido pleurico risultati sempre negativi agli esami colturali. II versamento pleurico è stato verosimilmente attribuito alla grave disfunzione sisto-diastolica. Dopo la risoluzione del quadro cardiovascolare, con un aumento della frazione di eiezione (FE 40\%), si è assistito a un progressivo miglioramento clinico e soggettivo, pur persistendo le numerose comorbidità.

A quattro mesi dalla dimissione ospedaliera, il paziente ha improvvisamente manifestato caratteristiche lesioni cutanee di tipo vescicoloso a carico della regione sinistra del collo, del torace e della spalla, associate a intenso dolore urente (Fig. 1). 


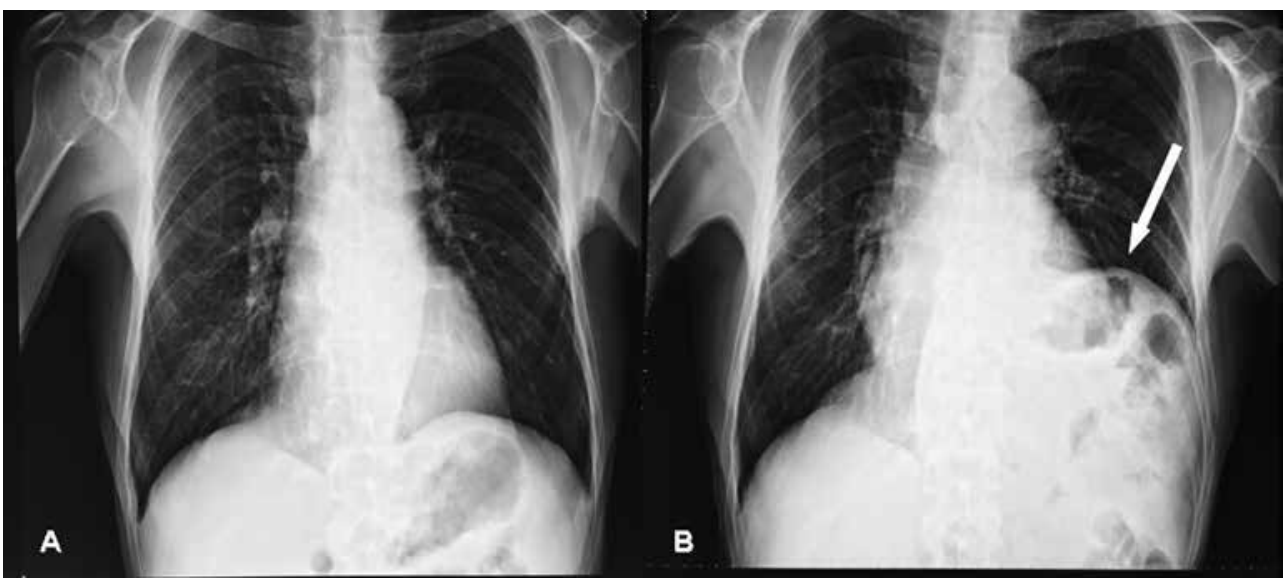

Fig. 2 - (A) Radiografia del torace, eseguita 15 giorni prima della comparsa del rash cutaneo che mostra la normalità degli emidiaframmi (B). Radiografia del torace, eseguita dopo 3 mesi dall'insorgenza dell'Herpes Zoster, che evidenzia la sopraelevazione dell'emidiaframma sinistro (freccia).

È stata diagnosticata un'infezione da Virus Herpes Zoster, riguardante il dermatomero C3-C4 di sinistra, e iniziata una terapia con Acyclovir a dosaggio adeguato alla funzione renale $(800 \mathrm{mg}$ per os, somministrati a fine dialisi e dopo 12 ore, per 10 giorni). Tale trattamento ha determinato un miglioramento progressivo delle lesioni cutanee e una parziale regressione della sintomatologia algica. In assenza di sintomi di disfunzione respiratoria riferiti dal paziente, nel corso di un controllo radiografico del torace eseguito a circa 3 mesi dall'insorgenza delle lesioni cutanee, viene evidenziata una netta sopraelevazione dell'emidiaframma sinistro, non rilevata a un radiogramma effettuato 15 giorni prima dell'infezione virale (Fig. 2). L'esame obiettivo toracico ha evidenziato un'area di ottusità in campo medio-basale sinistro e una riduzione omolaterale del murmure vescicolare; non erano, inoltre, rilevabili movimenti paradossi della parete addominale in posizione supina. La TC torace con mdc, eseguita allo scopo di evidenziare eventuali origini neoplastiche della lesione del diaframma, ha confermato il reperto della paralisi emidiaframmatica escludendo cause locali di compressione del nervo frenico $a b$ estrinseco. Non è stata eseguita la fluoroscopia per la valutazione di un eventuale movimento paradosso del diaframma (sniff test), in quanto il referto della TC torace era stato sufficientemente esaustivo.

Sulla base dei dati clinici, radiologici e temporali, l'elevazione dell'emidiaframma è stata verosimilmente attribuita all'infezione da VZV riguardante i dermatomeri C3-C4 e il nervo frenico.

\section{Discussione}

Il presente articolo descrive un raro caso di paralisi emidiaframmatica sinistra associata a infezione da Virus Varicella Zoster (VZV) nell'area del dermatomero omolaterale C3-C4. Il paziente ha manifestato l'eruzione a distribuzione dermatomerica unilaterale e la nevralgia posterpetica, tipicamente osservate nel corso dell'infezione da Herpes Zoster. L'elevazione emidiaframmatica sinistra non era presente a una radiografia del torace eseguita 15 giorni prima della comparsa dell'eruzione cutanea e, in totale assenza di una sintomatologia respiratoria, si è evidenziata dopo 3 mesi dall'esordio della malattia, nel corso di un controllo radiologico. La paralisi emidiaframmatica è spesso svelata incidentalmente da una radiografia del torace ottenuta, come nel nostro caso, per altri motivi.

La lesione del nervo frenico, da stiramento o da raffreddamento, è di comune riscontro durante gli interventi di cardiochirurgia. Altre cause meno frequenti di paralisi emidiaframmatica vengono individuate nella spondilosi cervicale, nella poliomielite, nei tumori compressivi del mediastino, nell'infiltrazione maligna da carcinoma bronchiale, nella polmonite, nella neuropatia diabetica, nell'embolizzazione iatrogena, nelle neuropatie (infettive e immuno-mediate), nelle vasculiti e nei traumi da incannulazione della vena succlavia (17).

Un caso di paralisi emidiaframmatica causata da Herpes Zoster è stato descritto per la prima volta da Halpern e Covner nel 1949 (5). Dalla letteratura finora pubblicata e disponibile sul motore di ricerca Pubmed, i casi di paralisi emidiaframmatica da VZV sono estremamente limitati e, dalla loro analisi, emergono alcune caratteristiche comuni: oltre l'85\% dei casi aveva più di 55 anni e le lesioni cutanee e la paralisi emidiaframmatica erano omolaterali nel $100 \%$ dei casi, il $75 \%$ dei quali presentava dispnea da sforzo, il $40 \%$ debolezza muscolare e il $25 \%$ non presentava alcun sintomo respiratorio associato; oltre il $20 \%$ dei casi riferiva una storia pregressa di patologie respiratorie (bronchiti, asma bronchiale, enfisema, neoplasie) e, in oltre il $70 \%$ dei casi, la paralisi emidiaframmatica era permanente.

Diversi studi hanno dimostrato che l'età è il più importante fattore di rischio per lo sviluppo dell'Herpes Zoster e della nevralgia posterpetica $(18,19)$ con la maggiore incidenza (5-10 casi su 1000) in individui nella sesta-ottava decade di vita; anche i pazienti con paralisi emidiaframmatica da Herpes Zoster tendono ad avere un'età avanzata.

Sono stati riportati in letteratura tre casi di paralisi diaframmatica unilaterale con una storia di insorgenza acuta o 
subacuta di dolore al collo o alla spalla senza lesioni cutanee (Zoster sine herpete) (20). Tutti e tre i casi sono stati empiricamente trattati con terapia antivirale (Valacyclovir $1000 \mathrm{mg}$ $\times 2 /$ die per 7 giorni) con conseguente riduzione della paralisi. Da una review della letteratura, effettuata da Oike nel 2012 in merito alla pubblicazione dei casi di paralisi emidiaframmatica associati a Herpes Zoster (21), risulta che la terapia antivirale è stata somministrata solo in $5 / 29$ casi e in un caso soltanto si è assistito alla remissione completa della paralisi. Lo stesso Autore descrive il case report di un paziente al quale era stato somministrato Famciclovir per 7 giorni al dosaggio di $750 \mathrm{mg}$ al giorno (la dose raccomandata di Famciclovir è da 500 a $1000 \mathrm{mg}$ al giorno per un eGFR da 10 a $50 \mathrm{~mL} / \mathrm{min}$ ), senza risoluzione della paralisi, ipotizzando la correlazione tra il sottodosaggio del farmaco antivirale e lo sviluppo della paralisi diaframmatica.

II nostro paziente è stato trattato con Acyclovir alla dose di $800 \mathrm{mg}$ per os 2 volte al giorno per 10 giorni, adeguando il dosaggio al grado di funzione renale (stadio 5D K/DIGO) (22, 23). È ben noto, infatti, che, nel soggetto uremico, dosaggi più elevati di Acyclovir possano esporre i pazienti a gravi complicanze neurologiche (agitazione, vertigini, allucinazioni, sonnolenza, letargia, tremori, confusione mentale, convulsioni o coma) $(24,25)$; tuttavia, in alcuni pazienti dializzati, sono stati osservati effetti collaterali neurologici (che sono, per ciascun paziente, dose-dipendenti) anche al dosaggio consigliato di $800 \mathrm{mg} \times 2 /$ die. Addirittura, un dibattito su questo argomento, tenutosi nella Mailing List SIN nel Maggio del 2004, portò a un articolo sul GIN (22) che arrivava a consigliare posologie addirittura inferiori rispetto a quelle comunemente utilizzate (dose di carico di $400 \mathrm{mg}$ seguita da una dose di mantenimento di $200 \mathrm{mg} \times 2 /$ die più eventuale dose supplementare di $400 \mathrm{mg}$ a fine dialisi) (26).

Nel nostro caso, ci siamo chiesti se lo schema terapeutico adottato (rispetto alla posologia di Acyclovir di $800 \mathrm{mg}$ x 5/die prevista per soggetti con normale funzione renale) potesse aver contribuito, analogamente a quanto suggerito da Oike, all'interessamento delle fibre motorie e se un differente dosaggio o un prolungamento della terapia antivirale a basse dosi avrebbero potuto evitare la paralisi del nervo frenico. Tuttavia, non vi è alcuna evidenza in merito al fatto che un aumento della posologia dell'Acyclovir (quando è già in atto la riattivazione del virus) sia in grado di proteggere dall'eventuale interessamento delle fibre motorie. Pertanto, in considerazione della prognosi relativamente benigna della paralisi emidiaframmatica, riteniamo che, sulla base delle attuali conoscenze nel paziente uremico, i rischi conseguenti agli incrementi posologici siano superiori rispetto agli eventuali benefici.

Infine, per quanto riguarda la profilassi, il vaccino per il Virus Varicella Zoster riduce il rischio di sviluppare la malattia ed è efficace nella prevenzione della nevralgia posterpetica (27), sebbene non vi siano prove che la vaccinazione protegga dalle sequele, inclusi i disordini neurologici.

\section{Conclusioni}

Abbiamo descritto un raro caso di paralisi emidiaframmatica conseguente a infezione da Virus Varicella Zoster (VZV) a distribuzione dermatomerica omolaterale, in un soggetto uremico. Nell'esaminare i pazienti con Herpes Zoster, in particolare i pazienti di età più avanzata e immunodepressi e quelli con una storia pregressa di malattie respiratorie, il Clinico dovrebbe essere consapevole della possibilità che l'Herpes Zoster a carico del dermatomero C3-C4-C5 possa complicarsi con il coinvolgimento del nervo frenico e il conseguente sviluppo della paralisi emidiaframmatica omolaterale, che può manifestarsi con dispnea da sforzo, debolezza muscolare o, come nel nostro caso, in maniera del tutto asintomatica. Un'aumentata consapevolezza da parte dei Medici riguardo alla capacità del VZV di danneggiare le fibre motorie può essere importante, soprattutto allo scopo di evitare procedure o diagnosi non corrette quando quadri clinici neurologici compatibili dovessero insorgere in rapporto temporale (da pochi giorni prima a poche settimane dopo) con la comparsa delle caratteristiche vescicole cutanee.

Ulteriori studi, in particolare nel soggetto uremico, sarebbero necessari allo scopo di valutare se differenti schemi posologici dei farmaci antivirali possano avere effetti protettivi sull'insorgenza della paralisi diaframmatica o, più in generale, sull'interessamento delle componenti motorie.

\section{Disclosures}

Financial support: No financial support was received for this submission.

Conflict of interest: The authors have no conflict of interest.

\section{Bibliografia}

1. Haanpää M, Häkkinen V, Nurmikko T. Motor involvement in acute herpes zoster. Muscle Nerve. 1997;11:1433-8.

2. Gibson GJ. Diaphragmatic paresis: pathophysiology, clinical features, and investigation. Thorax. 1989;44:960-70.

3. Wharton $\mathrm{M}$. The epidemiology of varicella-zoster virus infections. Infect Dis Clin North Am. 1996;10:571.

4. Kost RG, Strauss SE. Postherpetic neuralgia: pathogenesis, treatment, and prevention. N Engl J Med. 1996;335:32.

5. Halpern SL, Covner AH. Motor manifestation of herpes zoster: report of a case of associated permanent paralysis of the phrenic nerve. Arch Intern Med. 1949;84:907-16.

6. Spiers AS. Herpes zoster and its motor lesions, with a report of a case of phrenic paralysis. Med J Aust 1963;50:850-3.

7. Brostoff J. Diaphragmatic paralysis after herpes zoster. Br Med J. 1966;2:1571-2.

8. Anderson JP, Keal EE. Cervical herpes zoster and diaphragmatic paralysis. Br J Dis Chest. 1969;63:222-6.

9. Dutt AK. Diaphragmatic paralysis caused by herpes zoster. Am Rev Resp Dis. 1970;101:755-8.

10. Shivalingappa G. Diaphragmatic paralysis following herpes zoster. Gerontol Clin (Basel). 1970;12:283-7.

11. Derveaux L, Laquet LM. Hemidiaphragmatic paresis after cervical herpes zoster. Thorax. 1982;37:870-1.

12. Stowasser M, Cameron J, Oliver WA. Diaphragmatic paralysis 
following cervical herpes zoster. Med J Aust. 1990;153:555-6.

13. Soler JJ, Perpina M, Alfaro A. Hemidiaphragmatic paralysis caused by cervical herpes zoster. Respiration. 1996;63:403-6.

14. Paudyal BP, Karki A, Zimmerman M, Kayastha G, Acharya P. Hemidiaphragmatic paralysis: a rare complication of cervical herpes zoster. Kathmandu Univ Med J. 2006;4:246-8.

15. Hoque R, Schwendimann RN, Liendo C, Chesson AL Jr. Brachial neuritis with bilateral diaphragmatic paralysis following herpes zoster: a case report. J Clin Neuromusc Dis. 2008;9:402-6.

16. Bahadir C, Kalpakcioglu AB, Kurtulus D. Unilateral diaphragmatic paralysis and segmental motor paresis following herpes zoster. Muscle Nerve. 2008;38:1070-3.

17. Wilcox PG, Pardy RL. Diaphragmatic weakness and paralysis. Lung. 1989;167:323-41.

18. Harpaz R, Ortega-Sanchez IR, Seward JF. Prevention of herpes zoster: recommendation of the Advisory Committee on Immunization Practices (ACIP). MMWR Recomm Rep. 2008;57(RR-5):1-30.

19. Choo PW, Galil K, Donahue JG, Walker AM, Spiegelman D, Platt R. Risk factors for postherpetic neuralgia. Arch Intern Med. 1997;157:1217.

20. Crausman RS, Summerhill EM, McCool FD. Idiopathic diaphragmatic paralysis: Bell's Palsy of the diaphragm? Lung. 2009;187:153-7.
21. Oike $\mathrm{M}$, Naito $\mathrm{T}$, Tsukada $\mathrm{M}$, et al. A case of diaphragmatic paralysis complicated by herpes-zoster virus infection. Intern Med. 2012;51(10):1259-63.

22. D'Amico M, Fraticelli $M$, Limido A. Sulla mailing list: uso dell'acyclovir nei pazienti dializzati. [On the mailing list: use of acyclovir in patients on hemodialysis]. G Ital Nefrol. 2005;22(1):66-9.

23. Mesar I, Basić-Jukić N, Hudolin T, Katalinić L, Kes P. Varicella zoster virus reactivation in hemodialysis patients: manifestations, treatment, complications and outcome. Acta Clin Croat. 2011;50(4):549-52.

24. Arndt KA. Adverse reactions to acyclovir: topical, oral, and intravenous. J Am Acad Dermatol. 1988;18:188-90.

25. Fukunishi I, Inada T, Horie Y. Manic symptoms caused by acyclovir in a hemodialysis patient. Nephron. 1994;67:494.

26. Almond MK, Fan S, Dhillon S, Pollock AM, Raftery MJ. Avoiding acyclovir neurotoxicity in patients with chronic renal failure undergoing haemodialysis. Nephron. 1995;69:428-32.

27. Oxman MN, Levin MJ, Johnson GR, et al. A vaccine to prevent herpes zoster and postherpetic neuralgia in older adults. N Engl J Med. 2005;352: 2271-84.

\section{TEST DI VERIFICA}

1. La paralisi del nervo frenico è una condizione che raramente può complicare un'infezione da Herpes Zoster coinvolgente i dermatomeri:
a) $\mathrm{C} 1-\mathrm{C} 2$
b) $\mathrm{C} 3-\mathrm{C} 4-\mathrm{C} 5$
c) $\mathrm{T} 1-\mathrm{T} 3$
d) T3-T4-T5

2. Le cause più comuni di paralisi del nervo frenico sono:
a) Chirurgiche e traumatiche
b) Neoplastiche
c) Degenerative
d) Tutte le opzioni sono corrette

3. La paralisi emidiaframmatica può manifestarsi clinicamente:
a) Con dispnea da sforzo
b) Con debolezza muscolare
c) In modo del tutto asintomatico
d) Tutte le opzioni sono corrette

4. Nel trattamento dell'Herpes Zoster, il dosaggio orale dell'Acyclovir, raccomandato nel soggetto uremico in trattamento emodialitico, è:
a) $200 \mathrm{mg} \times 5 / \mathrm{die}$
b) $400 \mathrm{mg} \times 2 / \mathrm{die}$
c) $800 \mathrm{mg} \times 2 / \mathrm{die}$
d) $800 \mathrm{mg} \times 5 / \mathrm{die}$ 